\title{
Graves' Ophthalmopathy as an Indication Increased the Risk of Hypoparathyroidism After Bilateral Thyroidectomy
}

\author{
Kai-Pun Wong • Brian Hung-Hin Lang
}

Published online: 20 August 2011

(c) The Author(s) 2011. This article is published with open access at Springerlink.com

\begin{abstract}
Background Studies have evaluated the effect of thyroidectomy on the course of Graves' ophthalmopathy (GO) but it is unclear how GO as an indication might affect surgical outcomes. We aimed to evaluate the impact of this indication on surgical outcomes in Graves' disease (GD). Methods From 1995 to 2008, 329 patients with GD underwent thyroidectomy. Patients were stratified into two groups, namely, those with GO as indication (GO) and those with non-GO indication (non-GO). Outcomes were compared between the groups and outcomes with significance were further analyzed by multivariate analyses to determine independent factors.

Results The GO group was significantly older $(P<$ $0.001)$, had more males $(P<0.001)$, and fewer relapses $(P<0.001)$ than the non-GO group. It also had a higher proportion of total/near-total thyroidectomy $(P<0.001)$, despite a shorter operating time $(P=0.024)$ and less blood loss $(P=0.010)$. When only total/near-total thyroidectomy was considered, the GO group had significantly more permanent hypoparathyroidism than the non-GO group (9.2 vs. $1.6 \%, P=0.038$ ), but the rate of permanent hypoparathyroidism was similar in the two groups when only those with parathyroid autotransplantation were considered. Other complications were similar between the two groups. By multivariate analysis, GO as indication was an independent risk factor for temporary (OR 1.97,
\end{abstract}

K.-P. Wong · B. H.-H. Lang ( $ه)$

Division of Endocrine Surgery, Department of Surgery,

The University of Hong Kong, Queen Mary Hospital,

Pokfulam Road, Hong Kong, Hong Kong

e-mail: blang@hkucc.hku.hk

K.-P. Wong

e-mail: benwongkp@gmail.com
$P=0.033)$ and permanent hypoparathyroidism (OR 4.76, $P=0.007)$.

Conclusion $\mathrm{GO}$ as a surgical indication (i.e., unstable or active GO requiring ophthalmic treatment or follow-up) was associated with increased risk of temporary and permanent hypoparathyroidism after bilateral thyroidectomy. Routine parathyroid autotransplantation may reduce the risk of permanent hypoparathyroidism in this select patient group.

\section{Introduction}

Graves' disease (GD) is an autoimmune thyroid disease with an unpredictable clinical course. It is caused by thyroid receptor antibodies that activate the thyrotrophin (TSH) receptor leading to stimulation of cyclic adenosine monophosphate synthesis and production of thyroid hormones in the follicular cells [1, 2]. Treatment options include antithyroid drugs (ATD), radioactive iodine therapy (RAI), and thyroidectomy. In Europe and Asia, ATD is the first-line therapy, with RAI and thyroidectomy reserved as second-line in case of relapse, whereas in the United States, ATD is commonly used as an adjunct before RAI or thyroidectomy [3-5]. Our institution has adopted the former approach with satisfactory surgical outcomes [6]. However, the actual decision for RAI or thyroidectomy in relapsed cases remains controversial and depends on the presence of absolute or relative surgical indication(s). Graves' ophthalmopathy (GO) is one of the accepted indications but is considered a relative rather than an absolute indication for thyroidectomy. Advantages of thyroidectomy over RAI in GO include a rapid and durable control of hyperthyroidism and a potential for reversing proptosis, eyelid width, and diplopia [7]. Furthermore, RAI 
is known to worsen GO, particularly when glucocorticoids are omitted [8]. Although numerous studies have demonstrated the potential benefits of thyroidectomy in GO [7, 911], GO remains a relative surgical indication and it is still unclear how GO as an indication might affect surgical outcomes in GD. Most would presume that surgical outcomes are similar to other non-GO indications as the same procedure is performed. However, there is emerging evidence that patients with GO tend to have more active glandular disease and are more likely to relapse [12, 13]. Furthermore, one study has found that patients with severe GO are significantly more likely to suffer from both temporary and permanent hypoparathyroidism after bilateral thyroidectomy than those without GO $(P=0.004)$, although the exact mechanism remains unexplained [13]. The aim of this study was to evaluate the impact of GO as an indication relative to other accepted indications on the surgical outcomes of thyroidectomy for GD. Primary outcomes measured included recurrent laryngeal nerve injury, hypoparathyroidism, postoperative bleeding, and wound infection. Perhaps a better understanding might alter our decision on the extent of thyroid resection, intraoperative strategy, and perioperative management in this select group of patients.

\section{Patients and methods}

From January 1995 to December 2008, 329 consecutive patients undergoing thyroidectomy for GD were reviewed and analyzed. Surgical techniques and perioperative management remained similar regardless of surgical indication. The main indication or reason for choosing surgery over RAI was recorded. Patients with symptoms or signs of GO were assessed by independent ophthalmic assessment. Only those with unstable or active GO requiring ophthalmic treatment or follow-up before thyroidectomy were regarded as having GO as a surgical indication. Those with stable or inactive GO not requiring treatment or follow-up were not regarded as having $\mathrm{GO}$ as a surgical indication and their indication was categorized in the same way as those with no GO. If a patient had no clear surgical indication but expressed preference for surgery over RAI, it was regarded as patient preference. However, if a patient had no indication and did not express preference for surgery, it was regarded as undocumented.

Table 1 shows the baseline patient characteristics and surgical indications. There were $57(17.3 \%)$ males and 272 $(82.7 \%)$ females. The median (range) age at operation was 30 years (14-75). Eight (2.4\%) patients had previous radioiodine therapy before thyroidectomy. Seventy-six $(23.1 \%)$ patients had GO as surgical indication. Among the non-GO indications, patient preference $(33.7 \%)$ was the most common.
Table 1 Baseline patient characteristics and surgical indications (No. of patients $=329$ )

\begin{tabular}{ll}
\hline Age at operation (years) & $30(14-75)$ \\
Gender & $57(17.3)$ \\
Male & $272(82.7)$ \\
Female & $8(2.4)$ \\
Previous RAI therapy prior to surgery & $1(0-5)$ \\
Number of relapses prior to surgery & $18(0-90)$ \\
Duration of antithyroid medications (months) & $72(6-169)$ \\
Duration of follow-up (months) since surgery & \\
Surgical indications & $76(23.1)$ \\
Graves' ophthalmopathy & \\
Non-Graves' ophthalmopathy indications & $111(33.7)$ \\
Patient preference & $48(14.6)$ \\
Patient refusal for radioiodine therapy & $48(14.6)$ \\
Large goiter with pressure symptoms & $16(4.9)$ \\
Suspicious of malignancy & $23(7.0)$ \\
Young age/planning pregnancy & $7(2.1)$ \\
Undocumented &
\end{tabular}

Continuous data are expressed as median with range in parenthesis, unless otherwise indicated

The treatment strategy for GD remained the same throughout the entire study period. The first-line treatment was 18 months of ATD (either carbimazole or propylthiouracil). Beta-blocker agents were used for symptomatic control. A relapse was defined as a recurrence of hyperthyroidism after 18 months of ATD. Relapsed patients were given the option of RAI or surgery unless surgery was absolutely indicated, such as for those with suspected malignancy or pressure symptoms. To evaluate the effect GO as an indication on surgical outcomes in GD, patients were stratified into two groups, GO group (patients with GO as surgical indication, $n=76$ ) and non-GO group (patients with surgical indication other than GO, $n=253$ ) and their surgical outcomes were compared.

For preoperative preparation, all patients were prescribed ATD to achieve euthyroidism before surgery. Betablocker agents were prescribed for symptomatic control. Lugol solution was not routinely administered. Subtotal thyroidectomy was the preferred procedure before the year 2000. After that, total thyroidectomy became the preferred procedure. Reasons for the change in surgical approach were previously described [6]. Subtotal thyroidectomy was defined as resection that leaves up to 2-3 $\mathrm{g}$ of thyroid remnant on either one side or both sides and near-total thyroidectomy was resection that leaves less than $1 \mathrm{~g}$ of remnant behind. Surgical techniques and postoperative care had been previously described [14]. In brief, thyroidectomy was performed through a collar incision with the patient under general anesthesia and with endotracheal intubation. The strap muscles were separated in the midline and 
retracted laterally. Both recurrent laryngeal nerves (RLNs) and parathyroid glands were routinely identified and preserved. Parathyroid glands that were inadvertently removed or devascularized were immediately minced and autoimplanted into the ipsilateral sternocleidomastoid muscle. A wound drain was inserted selectively at the discretion of the operating surgeon.

Serum calcium and phosphate levels were measured within $6 \mathrm{~h}$ and every $12 \mathrm{~h}$ after surgery until stabilization. Calcium supplements with or without vitamin D analog were prescribed for those with symptomatic hypocalcemia or a serum calcium level $<1.70 \mathrm{mmol} / \mathrm{l}$ (normal range $=2.11-2.55 \mathrm{mmol} / \mathrm{l})$. Those who could discontinue calcium plus vitamin $\mathrm{D}$ analog supplement in the presence of normocalcemia within 12 months after surgery were categorized as having temporary hypoparathyroidism and those who had to continue for $>12$ months and had a below-normal serum parathyroid hormone level (normal range $=11-54 \mathrm{pg} / \mathrm{ml}$ ) were categorized as having permanent hypoparathyroidism. Oral thyroxine supplement was given on the day of discharge for hormonal replacement depending on the age and body weight of the patient.

Perioperative direct laryngoscopy was performed routinely before and within 1 week after the operation to assess vocal cord function. RLN palsy lasting more than 6 months after thyroidectomy as documented by direct laryngoscopy was regarded as permanent. To calculate the transient and permanent RLN palsy rates, the number of nerves at risk was used. There was no patient with preoperative cord palsy or who needed the RLN intentionally sacrificed at operation. The total number of nerves at risk was 658 (i.e., $329 \times 2$ ). All patients were regularly followed up 2 weeks after surgery, every 3-6 months for first 2 years, and yearly thereafter. All relevant clinical, laboratory, radiologic, and perioperative data were collected prospectively and followup data were regularly updated in a computerized database.

\section{Statistics}

The statistical analysis was performed using the SPSS ver. 18.0 (SPSS, Inc., Chicago, IL, USA). A $\chi^{2}$ test and Fisher's exact test were used for comparison of dichotomous variables, and the Mann-Whitney $U$ test was used for comparison of continuous variables between the GO and non$\mathrm{GO}$ groups. Outcomes that were significant were further analyzed in a multivariate analysis by logistic regression to determine independent risk factors. To improve clinical utility, before entering into the multivariate analysis, significant continuous variables were converted into binary variables using the overall median value as the cutoff. A binary logistic regression analysis was used in multivariate analysis in order to identify independent factors. A $P$ value $<0.05$ was considered statistically significant.

\section{Results}

Table 2 shows a comparison of patient demographics, operative findings, type of resection, and histological findings between the GO and non-GO groups. The GO group was older in median age (36 vs. 30 years, $P<0.001)$ and had a greater proportion of males than the non-GO group (32.9 vs. $12.6 \%, P<0.001$ ). However, the GO group had significantly fewer relapses prior to surgery than the non-GO group $(0$ vs. $1, P<0.001)$. There was a greater proportion of patients who underwent total or neartotal thyroidectomy in the GO group (80.3 vs. $59.7 \%$, $P=0.001$ ), but the volume of blood loss was less (50 vs. $80 \mathrm{ml}, P=0.010)$ and the operating time was shorter in the GO group (139 vs. $150 \mathrm{~min}, P=0.024$ ). The excised gland was lighter in the GO group (46 vs. $54 \mathrm{~g}$, $P=0.004)$. There were a greater number of parathyroid glands identified per procedure in the GO group (4 vs. 3 , $P=0.023)$, but the number of glands transplanted and rate of transplantation appeared similar between the groups. Histological findings were similar for the two groups.

Table 3 shows a comparison of short- and long-term surgical outcomes between the GO and non-GO groups. There was significantly more temporary hypoparathyroidism (32.9 vs. $20.2 \%, P=0.021)$ and permanent hypoparathyroidism ( 9.2 vs. $2.8 \%, P=0.015)$ in the $\mathrm{GO}$ group. When only total or near-total versus subtotal thyroidectomy was considered, the GO group was significantly more likely to suffer permanent hypoparathyroidism than the non-GO group $(9.2$ vs. $1.6 \%, P=0.038)$. When patients were stratified according to whether parathyroid autotransplantation was performed, the rate of permanent hypoparathyroidism was significantly higher in the GO group than in the non-GO group when parathyroid autotransplantation was not performed $(P=0.032)$, whereas the rate was similar when parathyroid autotransplantation was performed $(P=0.338)$. There was no significant difference in other surgical complications, including vocal cord palsy, postoperative bleeding, postoperative infection, and length of hospital stay. In the subtotal thyroidectomy group, after a median follow-up of 107.2 months, there were $10(8.5 \%)$ patients who suffered persistent or recurrent GD; 5 required additional RAI therapy while the other 5 decided to resume ATD. Two of the five patients who received RAI eventually became hypothyroid and required thyroxine replacement. In the total or near-total thyroidectomy group, after a median follow-up of 48.7 months, no patient developed recurrent GD or required ATD or RAI. Therefore, the overall rate of persistent or recurrent GD in subtotal thyroidectomy was $10 / 117(8.5 \%)$. Of the 117 subtotal thyroidectomies performed, only 23 (19.7\%) patients maintained normal thyroid function not requiring daily thyroxine replacement therapy. 
Table 2 A comparison of patient demographics, operative findings, type of resection, and histological findings between patients with Graves' ophthalmopathy as indication (GO group) and patients with other indications (non-GO group)

Continuous data are expressed as median with range in parenthesis, unless otherwise indicated

GO Graves' ophthalmopathy, FTC follicular thyroid cancer, $P T C$ papillary thyroid cancer

Bold indicates $P$ value $<0.05$ as statistical significance

Table 3 A comparison of primary postsurgical outcomes between patients with Graves' ophthalmopathy as indication (GO) and those with other nonGO indications (non-GO)

Continuous data are expressed as median with range in parenthesis, unless otherwise indicated

${ }^{\text {a }}$ Calculated based on total number of nerves at risk

Bold indicates $P$ value $<0.05$ as statistical significance

\begin{tabular}{lllr}
\hline & GO $(n=76)$ & Non-GO $(n=253)$ & $P$ value \\
\hline Age at operation (years) & $36(18-75)$ & $30(14-73)$ & $<\mathbf{0 . 0 0 1}$ \\
Gender (male:female) & $25: 51$ & $32: 221$ & $<\mathbf{0 0 0 1}$ \\
Family history & $11(16.7)$ & $51(23.7)$ & 0.190 \\
Previous radioiodine therapy & $3(3.9)$ & $5(2.0)$ & 0.328 \\
Number of relapses & $0(0-5)$ & $1(1-5)$ & $<\mathbf{0 . 0 0 1}$ \\
0 & $34(44.7)$ & $38(15.0)$ & \\
1 & $17(22.4)$ & $74(29.2)$ & \\
$\geq 2$ & $16(21.1)$ & $104(41.1)$ & $\mathbf{0 . 0 2 4}$ \\
Operating time (min) & $139(75-215)$ & $150(60-360)$ & $\mathbf{0 . 0 1 0}$ \\
Blood loss (ml) & $50(0-300)$ & $80(0-140)$ & $\mathbf{0 . 0 0 4}$ \\
Weight of excised gland (g) & $46(6-240)$ & $54(6-727)$ & $\mathbf{0 . 0 0 1}$ \\
Type of resection & & & \\
Subtotal thyroidectomy & $15(19.7)$ & $102(40.3)$ & \\
Total or near-total thyroidectomy & $61(80.3)$ & $151(59.7)$ & $\mathbf{0 . 0 2 3}$ \\
No. of parathyroid glands identified per operation & $4(0-4)$ & $3(0-4)$ & 0.876 \\
No. of parathyroid glands autotransplanted per operation & $1(0-2)$ & $1(0-3)$ & 0.990 \\
Parathyroid autotransplantation & $37(50.7)$ & $126(50.6)$ & 0.882 \\
Histological findings & & & 0.410 \\
Diffuse hyperplasia & $71(93.4)$ & $245(96.8)$ & $7(2.8)$ \\
Diffuse hyperplasia with occult PTC & $4(5.3)$ & $1(0.4)$ & $20(15.0)$ \\
Diffuse hyperplasia with occult FTC & $1(1.3)$ & $7(13.5)$ & \\
Concomitant autoimmune thyroiditis & & & \\
\hline
\end{tabular}

\begin{tabular}{lccc}
\hline Postoperative outcome & GO $(n=76)$ & Non-GO $(n=253)$ & $P$ value \\
\hline Hospital stay (days) & $3(1-7)$ & $3(1-11)$ & 0.232 \\
Temporary hypoparathyroidism & $25(32.9)$ & $51(20.2)$ & $\mathbf{0 . 0 2 1}$ \\
Subtotal thyroidectomy & $0 / 15(0.0)$ & $21 / 102(20.6)$ & 0.629 \\
Total or near-total thyroidectomy & $25 / 61(41.0)$ & $30 / 151(19.9)$ & 0.073 \\
Permanent hypoparathyroidism & $7(9.2)$ & $7(2.8)$ & $\mathbf{0 . 0 1 5}$ \\
Subtotal thyroidectomy & $0 / 15(0.0)$ & $3 / 102(2.9)$ & 0.763 \\
Total or near-total thyroidectomy & $7 / 61(11.5)$ & $4 / 151(2.6)$ & $\mathbf{0 . 0 3 8}$ \\
Permanent hypoparathyroidism & $7(9.2)$ & $7(2.8)$ & \\
With parathyroid autotransplantation & $2 / 35(5.7)$ & $3 / 126(2.4)$ & 0.338 \\
Without parathyroid autotransplantation & $5 / 41(12.2)$ & $4 / 127(3.1)$ & $\mathbf{0 . 0 3 2}$ \\
Vocal cord palsy-temporary $^{\mathrm{a}}$ & $2(1.3)$ & $6(1.2)$ & 0.970 \\
Vocal cord palsy-permanent $^{\mathrm{a}}$ & $0(0.0)$ & $2(0.4)$ & 0.420 \\
Postoperative bleeding & $1(1.4)$ & $6(2.4)$ & 0.595 \\
Wound infection & $0(0.0)$ & $1(0.4)$ & 0.588 \\
\hline
\end{tabular}

To determine independent risk factors for postoperative hypoparathyroidism, age at operation, gender, number of relapses, type of resection, operating time $>150 \mathrm{~min}$, weight of gland $>52 \mathrm{~g}$, parathyroid autotransplantation, and GO as an indication were analyzed. Gender, type of operation, parathyroid autotransplantation and GO turned out to be significant in univariate analysis (not shown) but on multivariate analysis, only female gender (OR 2.77, 95\% CI: $1.14-6.73, P=0.024$ ), total or near-total thyroidectomy (OR 2.44, 95\% CI: $1.31-4.56, P=0.005$ ), and GO as indication (OR 1.97, 95\% CI: $1.06-3.66, P=0.033)$ turned out to be independent risk factors for temporary hypoparathyroidism (see Table 4). For permanent hypoparathyroidism, operating time $>150$ min (OR 10.02, 95\% CI: $2.65-37.91$, $P=0.001$ ) and GO as indication (OR 4.76, 95\% CI: $1.52-14.88, P=0.007)$ were independent risk factors (see Table 5). 
Table 4 A multivariate analysis of factors leading to temporary hypoparathyroidism after thyroidectomy in Graves' disease

\begin{tabular}{llll}
\hline & Odds ratio & $95 \%$ CI & $P$ value \\
\hline $\begin{array}{l}\text { Female gender } \\
\begin{array}{l}\text { Total or near-total } \\
\text { thyroidectomy }\end{array}\end{array}$ & 2.77 & $(1.14-6.73)$ & $\mathbf{0 . 0 2 4}$ \\
$\begin{array}{l}\text { Parathyroid autotransplantation } \\
\begin{array}{l}\text { Graves' ophthalmopathy as a } \\
\text { surgical indication }\end{array}\end{array}$ & 1.69 & $(1.31-4.56)$ & $\mathbf{0 . 0 0 5}$ \\
\hline
\end{tabular}

Bold indicates $P$ value $<0.05$ as statistical significance

Table 5 A multivariate analysis of factors leading to permanent hypoparathyroidism after thyroidectomy in Graves' disease

\begin{tabular}{lccc}
\hline & Odds ratio & $95 \%$ CI & $P$ value \\
\hline Operating time $>150$ min & 10.02 & $2.65-37.91$ & $\mathbf{0 . 0 0 1}$ \\
Graves' ophthalmopathy & 4.76 & $1.52-14.88$ & $\mathbf{0 . 0 0 7}$ \\
as surgical indication & & & \\
\hline
\end{tabular}

Bold indicates $P$ value $<0.05$ as statistical significance

\section{Discussion}

The general management of GD remains controversial. The issue of whether ATD, RAI, or surgery should be used has long been debated and the decision varies greatly depending on different parts of the world [3]. For those with relapsed GD after an adequate course of ATD, the decision for either RAI or thyroidectomy mostly depends on the presence of absolute or relative surgical indication. Thyroidectomy has gained an increasing role over RAI in relapsed GD as it offers more rapid and lasting control of the hyperthyroidism, and for those with preexisting GO, it could also possibly reverse a certain degree of proptosis, eyelid width, and diplopia [7, 9-11]. In contrast to previous studies that evaluated the effect of thyroidectomy on GO, the aim of the present study was to evaluate whether GO, which remains a relative surgical indication, would affect surgical outcomes in GD. We postulated that GO as an indication might adversely affect outcomes as the disease is generally more active and severe, making the surgery more technically demanding $[12,13]$. Furthermore, a study has found that patients with severe GO are more likely to suffer from hypoparathyroidism after thyroidectomy [13]. Since GO is common and is present in about a third of patients with GD [15], the present analysis regarded only patients with active or unstable GO requiring ophthalmic treatment or follow-up as having a genuine indication for thyroidectomy. Those with stable GO not requiring treatment or follow-up were not regarded as having GO as a surgical indication and their indications were categorized in the same way as those without GO.
The present analysis comprised two parts. The first part was to evaluate whether there were differences in outcomes between the GO and non-GO groups. Our data showed that the GO group had a significantly shorter operating time, less blood loss, and lighter excised thyroid gland weight. These findings are consistent with our previous observation that there was an increasing number of patients with GO undergoing thyroidectomy recently [16], and we believed the shorter operating time and less blood loss were a result of the surgical technical improvements and not a result of GO per se. The gland weight of the excised thyroid was lighter in the GO group because GO was a result of the autoimmune cross-activity between the thyroid and orbital antigens and not related to the gland size [13]. The data showed that the GO group was significantly more likely to suffer both temporary and permanent hypoparathyroidism after bilateral thyroidectomy than the non-GO group, even though the overall rate of hypoparathyroidism was compatible to those of other studies [17-19]. The risk of other complications was not significantly different between the two groups. When the extent of surgery (i.e., total or neartotal vs. subtotal thyroidectomy), a well-known factor for hypoparathyroidism [20], was adjusted between the two groups, the GO group still had a significantly higher risk of permanent hypoparathyroidism than the non-GO group (9.2 vs. $1.6 \%)$. This was despite the fact that there were a greater number of parathyroid glands identified in the GO group and a similar proportion of parathyroid autotransplantations performed at the time of operation in the two groups. Interestingly, both the number of parathyroid glands identified and parathyroid autotransplantation were shown to reduce the rate of permanent hypoparathyroidism in thyroidectomy [21-23]. This was supported by the subgroup analysis when patients were stratified according to whether parathyroid autotransplantation was performed or not. Our data showed that the permanent hypoparathyroidism rate was similar between the GO and non-GO groups when parathyroid autotransplantation was performed. In fact, the rate of permanent hypoparathyroidism was reduced from 12.2 to $5.7 \%$ in the GO group. To further determine if $\mathrm{GO}$ as a surgical indication was an independent factor for temporary and permanent hypoparathyroidism, two multivariate analyses were done. In concordance with the first part of the analysis, GO as a surgical indication turned out to be an independent factor for both temporary and permanent hypoparathyroidism. Female gender and extent of resection were the other independent risk factors for temporary hypoparathyroidism, and operating time $>150$ min was the other independent risk factor for permanent hypoparathyroidism. All of these factors, with the exception of operating time, were previously reported to be risk factors [21-25]. The authors believed the length of operating time was a possible 
surrogate marker of the degree of technical difficulty with the procedure.

There are a number of possible explanations for the higher incidence of hypoparathyroidism in GO after bilateral thyroidectomy. First, those with GO tend to have more active glandular disease than those without GO as indicated by the higher level of TSH-receptor auto-antibodies present $[26,27]$. This higher glandular activity could possibly translate into increased adhesions between the thyroid and parathyroids and microvessel density on the thyroid gland making the subcapsular dissection and preservation of the parathyroid glands and their blood supply technically more difficult for the surgeon. Since the number of parathyroid glands identified was actually higher in the GO group than in the non-GO group, it was not related to identifying the parathyroids but rather related to preserving the blood supply to the parathyroids. The second explanation for the higher incidence of hypoparathyroidism in GO might be related to the phenomenon of "hungry bone" syndrome after surgery as reported previously in GD after thyroidectomy [25]. Perhaps patients with active GO might have a more severe form of hungry bone syndrome. However, this would explain only the higher postoperative temporary hypoparathyroidism and not the permanent hypoparathyroidism. Perhaps a postoperative PTH level would help to distinguish hungry bone syndrome from temporary hypoparathyroidism.

We believe that a number of strategies could be adopted in the future to reduce hypoparathyroidism. First, for patients with GO undergoing thyroidectomy, perhaps less resection such as a subtotal thyroidectomy could be considered. In our subgroup analysis, it was interesting to note that no patient suffered permanent hypoparathyroidism after subtotal thyroidectomy in the GO group. However, one must also consider the long-term problem of relapse and delayed hypothyroidism associated with subtotal thyroidectomy [6]. The other strategy would be to increase the proportion of parathyroid autoimplantation. Instead of practicing selective parathyroid autoimplantation, which in our series amounted to $51 \%$, routine or $100 \%$ autoimplantation of at least one viable parathyroid gland during surgery might be a better strategy in patients with GO. Also, given the relatively high incidence of temporary hypoparathyroidism $(41.0 \%)$, patients with GO as a surgical indication undergoing a total thyroidectomy might perhaps benefit from routine administration of calcium and vitamin D supplementation to reduce the risk of symptomatic postoperative hypocalcemia.

The limitations of this study included the retrospective nature of data analysis and the fact that the data were collected for a 14-year period so there might have been subtle changes in referral pattern and practice contributing to the higher incidence of hypoparathyroidism [16]. Also, our study did not contain data on thyroid-stimulating hormone receptor (TSHR), which is an important marker for the severity of GD, so our proposal of more active disease in the GO group was based purely on our clinical and operative observations. Data on postoperative parathyroid hormone and vitamin D levels were not available and would have been useful in providing a better understanding of the cause of postoperative hypoparathyroidism. As vitamin D deficiency is prevalent in our geographical area, this could have also contributed to the rate of temporary hypoparathyroidism in the two groups [28]. Further studies focusing on the dynamics of vitamin $\mathrm{D}$ and its relation to hypoparathyroidism in GD and GO might prove fruitful. Also, it would have been interesting to evaluate any eye changes before and after total or near-total thyroidectomy or subtotal thyroidectomy in patients with active GO.

\section{Conclusion}

GO as a surgical indication (i.e., unstable or active GO requiring ophthalmic treatment or follow-up) was associated with increased risk of temporary and permanent hypoparathyroidism after bilateral thyroidectomy. Routine or $100 \%$ parathyroid autotransplantation may reduce the risk of permanent hypoparathyroidism in this select patient group.

Open Access This article is distributed under the terms of the Creative Commons Attribution Noncommercial License which permits any noncommercial use, distribution, and reproduction in any medium, provided the original author(s) and source are credited.

\section{References}

1. Nielsen CH, Brix TH, Leslie RG, Hegedus L (2009) A role for autoantibodies in enhancement of pro-inflammatory cytokine responses to a self-antigen, thyroid peroxidase. Clin Immunol 133(2):218-227

2. Prabhakar BS, Bahn RS, Smith TJ (2003) Current perspective on the pathogenesis of Graves' disease and ophthalmopathy. Endocr Rev 24(6):802-835

3. Stalberg P, Svensson A, Hessman O, Akerstrom G, Hellman P (2008) Surgical treatment of Graves' disease: evidence-based approach. World J Surg 32(7):1269-1277. doi:10.1007/s00 268-008-9497-9

4. Tominaga T, Yokoyama N, Nagataki S, Cho BY, Koh CS, Chen $\mathrm{JL}$ et al (1997) International differences in approaches to 131I therapy for Graves' disease: case selection and restrictions recommended to patients in Japan, Korea, and China. Thyroid 7(2): 217-220

5. Wartofsky L, Glinoer D, Solomon B, Nagataki S, Lagasse R, Nagayama $Y$ et al (1991) Differences and similarities in the diagnosis and treatment of Graves' disease in Europe, Japan, and the United States. Thyroid 1(2):129-135

6. Ku CF, Lo CY, Chan WF, Kung AW, Lam KS (2005) Total thyroidectomy replaces subtotal thyroidectomy as the preferred surgical treatment for Graves' disease. ANZ J Surg 75(7): $528-531$ 
7. Menconi F, Marino M, Pinchera A, Rocchi R, Mazzi B, Nardi M et al (2007) Effects of total thyroid ablation versus near-total thyroidectomy alone on mild to moderate Graves' orbitopathy treated with intravenous glucocorticoids. J Clin Endocrinol Metab 92(5):1653-1658

8. Leech NJ, Dayan CM (1998) Controversies in the management of Graves' disease. Clin Endocrinol (Oxf) 49(3):273-280

9. Tallstedt L, Lundell G, Torring O, Wallin G, Ljunggren JG, Blomgren $\mathrm{H}$ et al (1992) Occurrence of ophthalmopathy after treatment for Graves' hyperthyroidism The Thyroid Study Group. N Engl J Med 326(26):1733-1738

10. Abe Y, Sato H, Noguchi M, Mimura T, Sugino K, Ozaki O et al (1998) Effect of subtotal thyroidectomy on natural history of ophthalmopathy in Graves' disease. World J Surg 22(7):714-717. doi: $10.1007 / \mathrm{s} 002689900458$

11. Levitt MD, Edis AJ, Agnello R, McCormick CC (1988) The effect of subtotal thyroidectomy on Graves' ophthalmopathy. World J Surg 12(5):593-597. doi:10.1007/BF01655858

12. Eckstein AK, Lax H, Losch C, Glowacka D, Plicht M, Mann K et al (2007) Patients with severe Graves' ophthalmopathy have a higher risk of relapsing hyperthyroidism and are unlikely to remain in remission. Clin Endocrinol (Oxf) 67(4):607-612

13. Hassan L, Danila R, Maurer E, Osei-Agymang T, Zielke A (2008) Severe Graves' ophthalmopathy may be a factor for the development of postthyroidectomy hypocalcemia. Exp Clin Endocrinol Diabetes 116:614-618

14. Lang BH, Lo CY (2005) Total thyroidectomy for multinodular goiter in the elderly. Am J Surg 190(3):418-423

15. Bahn RS, Heufelder AE (1993) Pathogenesis of Graves' ophthalmopathy. N Engl J Med 329(20):1468-1475

16. Yip J, Lang BH, Lo CY (2011) Changing trend in surgical indication and management for Graves' disease. Am J Surg. doi: 10.1016/j.amjsurg.2011.01.029

17. Miccoli P, Vitti P, Rago T, Iacconi P, Bartalena L, Bogazzi F et al (1996) Surgical treatment of Graves' disease: subtotal or total thyroidectomy? Surgery 120(6):1020-1024; discussion 1024-1025

18. Palit TK, Miller CC 3rd, Miltenburg DM (2000) The efficacy of thyroidectomy for Graves' disease: a meta-analysis. J Surg Res 90(2):161-165

19. Witte J, Goretzki PE, Dotzenrath C, Simon D, Felis P, Neubauer M et al (2000) Surgery for Graves' disease: total versus subtotal thyroidectomy-results of a prospective randomized trial. World J Surg 24(11):1303-1311. doi:10.1007/s002680010216

20. Abboud B, Sargi Z, Akkam M, Sleilaty F (2002) Risk factors for postthyroidectomy hypocalcemia. J Am Coll Surg 195(4): $456-461$

21. Lo CY, Lam KY (2001) Routine parathyroid autotransplantation during thyroidectomy. Surgery 129(3):318-323

22. Thomusch O, Machens A, Sekulla C, Ukkat J, Brauckhoff M, Dralle H (2003) The impact of surgical technique on postoperative hypoparathyroidism in bilateral thyroid surgery: a multivariate analysis of 5846 consecutive patients. Surgery 133(2): $180-185$

23. Abboud B, Sleilaty G, Zeineddine S, Braidy C, Aouad R, Tohme $\mathrm{C}$ et al (2008) Is therapy with calcium and vitamin D and parathyroid autotransplantation useful in total thyroidectomy for preventing hypocalcemia? Head Neck 30(9):1148-1154; discussion $1154-1155$

24. Thomusch O, Machens A, Sekulla C, Ukkat J, Lippert H, Gastinger I et al (2000) Multivariate analysis of risk factors for postoperative complications in benign goiter surgery: prospective multicenter study in Germany. World J Surg 24(11):1335-1341. doi:10.1007/s002680010221

25. Yamashita H, Noguchi S, Tahara K, Watanabe S, Uchino S, Kawamoto H et al (1997) Postoperative tetany in patients with Graves' disease: a risk factor analysis. Clin Endocrinol (Oxf) 47(1):71-77

26. Gerding $\mathrm{MN}$, van der Meer JW, Broenink M, Bakker O, Wiersinga WM, Prummel MF (2000) Association of thyrotrophin receptor antibodies with the clinical features of Graves' ophthalmopathy. Clin Endocrinol (Oxf) 52(3):267-271

27. Eckstein AK, Plicht M, Lax H, Neuhauser M, Mann K, Lederbogen $S$ et al (2006) Thyrotropin receptor autoantibodies are independent risk factors for Graves' ophthalmopathy and help to predict severity and outcome of the disease. J Clin Endocrinol Metab 91(9):3464-3470

28. Lang BH, Lo CY (2010) Vitamin D3 deficiency is associated with late-onset hypocalcemia after minimally invasive parathyroidectomy in a vitamin D borderline area. World J Surg 34(6): 1350-1355. doi:10.1007/s00268-009-0377-8 